Communications in Physics, Vol. 26, No. 1 (2016), pp. 67-73

DOI:10.15625/0868-3166/26/1/7806

\title{
MEASURING ANTI-BUNCHING EFFECT FROM SINGLE DYE MOLECULES AND SINGLE QUANTUM DOTS
}

\author{
NGUYEN THI THANH BAO AND DINH VAN TRUNG ${ }^{\dagger}$ \\ Institute of Physics, Vietnam Academy of Science and Technology \\ DANG TUYET PHUONG \\ Institute of Chemistry, Vietnam Academy of Science and Technology \\ ${ }^{\dagger}$ E-mail: dvtrung@iop.vast.ac.vn \\ Received 02 February 2016
}

Accepted for publication 05 May 2016

\begin{abstract}
Antibunching is a quantum effect demonstrating clearly the quantum nature of the radiation field. Its detection through measurements of the second order correlation function is a direct proof of the presence of single molecule or single nano particle. In this paper we present the experimental setup of the Hanbury Brown - Twiss interferometer and the measurement results of the antibunching effect from single Rhodamine $B$ dye molecules and single CdTe quantum dots in dilute solution. By fitting the second order correlation data, we derive a fluorescence lifetime of approximately 2 ns for Rhodamine B and $45 \mathrm{~ns}$ for CdTe quantum dots. Our results demonstrate an alternative way for determining the fluorescence lifetime using the antibunching effect.
\end{abstract}

Keywords: quantum dot, Rhodamine, antibunching.

Classification numbers: 078.67.Hc, 07.60.Ly.

\section{INTRODUCTION}

Detection and characterization of single molecules or single nanoparticle have been studied intensively with applications in many different research fields such as nanoscience and nanotechnology, molecular biology etc. [1-12]. At single molecule or single nanoparticle level such as quantum dots the radiation field cannot be described satisfactorily in the classical sense and the its quantum properties become more apparent. Basically these emitters now behave as single photon sources and their characterization is most often investigated using second order correlation function or the photon antibunching effect measured with the Hanbury-Brown and Twiss (HBT) setup [1]. In a simple term, the photon antibunching effect represents the fact that an isolated quantum system such as single molecules or nanoparticles undergoing excitation-emission cycles can emit only one photon at a time. Therefore, for a quantum system with finite lifetime in the 
excited state, the second order intensity correlation function $\mathrm{g}^{(2)}(\tau)$ shows a dip, ideally should be zero, for time intervals comparable to the lifetime of the system. The photon antibunching behavior could be considered as a proof of the detection of single photon emitters, i.e. single molecule or single nanoparticle. This effect has been widely used to study the quantum statistics of the radiation field and to study single photon emitters. We should emphasize that this effect could be easily washed away by the presence of increasing number of emitters. Therefore, experimental measurement of this effect is very difficult and usually requires both sensitive instrumentation and careful sample preparation. In this paper we will review the basics of antibunching effect and describe our experimental setup to measure this effect and present the results for single dye molecules and single nanoparticles.

\section{PHOTON ANTIBUNCHING EFFECT}

Classical Maxwell's equations of electromagnetic theory can describe accurately the radiation field generated by fluorescence from an ensemble of emitters such as atoms or molecules. However, the quantization of the radiation field is required to explain the statistical properties of light under non classical conditions such as laser radiation or quantum correlations [1-4]. Single photon or the quantum of the radiation field behaves as a particle instead as wave in the classical theory. The difference between quantum and classical properties of light can most easily seen in the correlation between photon detection events. The experimental setup for measuring such correlation is the well known Hanbury Brown - Twiss interferometer as shown in Fig. 1 [1-6].

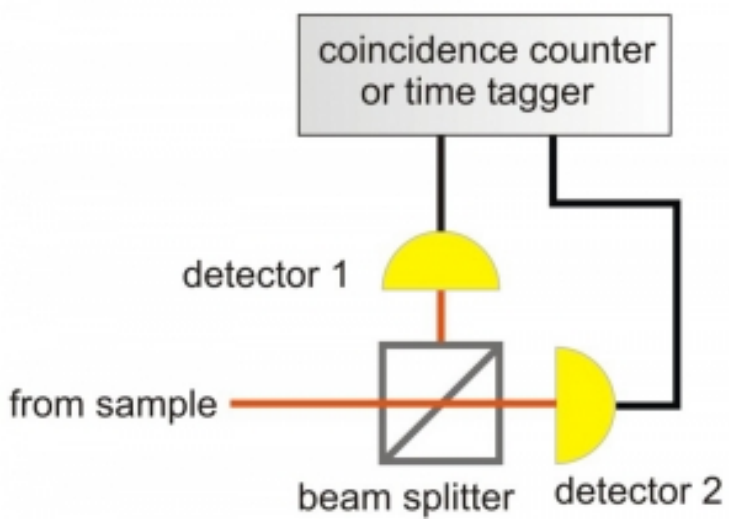

Fig. 1. Hanbury-Brown and Twiss interferometer setup for measuring second order intensity correlation $\mathrm{g}^{(2)}(\tau)$.

A beam of light is sent to a 50:50 beam splitter so that half of the intensity is reflected while the other half is transmitted. However, a single photon will need to choose either path 1 or path 2 with $50 \%$ probability when passes through the 50:50 beam splitter because it cannot split itself into two halves. The sensitive photon detectors will register the presence of that single photon in either 
path 1 or path 2 and the probability to produce simultaneous detection at the two photon detectors is expected to be zero. In other words, the single photon will never be bunched. For fluorescence photons emitted from single atoms or molecules, the HBT setup can be used to measure the second order correlation function $g^{(2)}(\tau)[1,13]$ :

$$
g^{(2)}(\tau)=1-\frac{1}{N} e^{-\left|\tau / \tau_{F}\right|}
$$

where $\tau$ is the time delay between the detection of photon in channel 1 and channel 2, $N$ is the effective number of emitters in the measurement volume, $\tau_{F}$ is the fluorescence lifetime of the emitters. Therefore, the correlation function $g^{(2)}(\tau)$ provides very useful information on the quantum properties of the radiation field emitted by the system and the internal properties such as fluorescence lifetime.

\section{INSTRUMENT DESCRIPTION}

To measure the antibunching effect, we adapted the previously developed Fluorescence Correlation Spectroscopy (FCS) with the Hanbury Brown - Twiss setup. The schematic sketch of our experiment setup is shown in Fig. 2. The excitation laser is a continuous wave diode pumped Nd:YAG laser emitting an average power of $40 \mathrm{~mW}$ at $532 \mathrm{~nm}$. The power level of the Nd:YAG is kept stable using a temperature controller based on a Peltier element. The excitation laser beam is then reduced to suitable level for our experiment using a set of neutral density filters. A spatial filter consisting of a pair of plano-convex lenses L1 and L2 together with a $20 \mu \mathrm{m}$ precision pin hole mounted on an accurate XYZ motion stage is used to clean the laser beam to TEM00 spatial mode in order to reach the diffraction limit at the focus spot of the laser beam on the sample. The clean excitation beam is then focused on the sample using a high numerical aperture oil immersion microscope objective (Edmund Optics with NA=1.25). Our sample is mounted on a nanopositioning stage developed in-house and capable of scanning in $\mathrm{X}$ and $\mathrm{Y}$ directions with $\sim 10 \mathrm{~nm}$ accuracy for optimizing the measurement position within the sample. The same microscope objective is used to collect the fluorescence light from the sample in a typical epifluorescence configuration. The fluorescence light is separated from the excitation laser beam using a dichroic mirror DM before being focused onto a $50 \mu \mathrm{m}$ pinhole by a plano-convex lens L3. A combination of long pass (BP) and band pass (IF) filters before the pinhole allow us to block the background including scattered laser light and any stray light from outside while leaving the fluorescence light to pass unhindered. The lens L4 recollimates the fluorescence light beam before sending it to the detection part. The Hanbury Brown - Twiss configuration is implemented in our setup using a polarizing beam splitter cube (Thorlabs) to split the incoming fluorescence light into two channels. Two photon counting avalanche photodiode (APD) operating in Geiger mode are used to detect the fluorescence signal in each channel. Photon pulses from the output of APD detectors are then correlated to form the $\mathrm{g}^{(2)}(\tau)$ function using Picoharp 300 module from PicoQuant (Germany). The silicon APD with high quantum efficiency, from about $60 \%$ in the red region to nearly $90 \%$ at the most sensitive wavelength of $850 \mathrm{~nm}$, allows a highly sensitive single photon detection in comparison to the previously used photomultiplier tube in our experiment setup. 


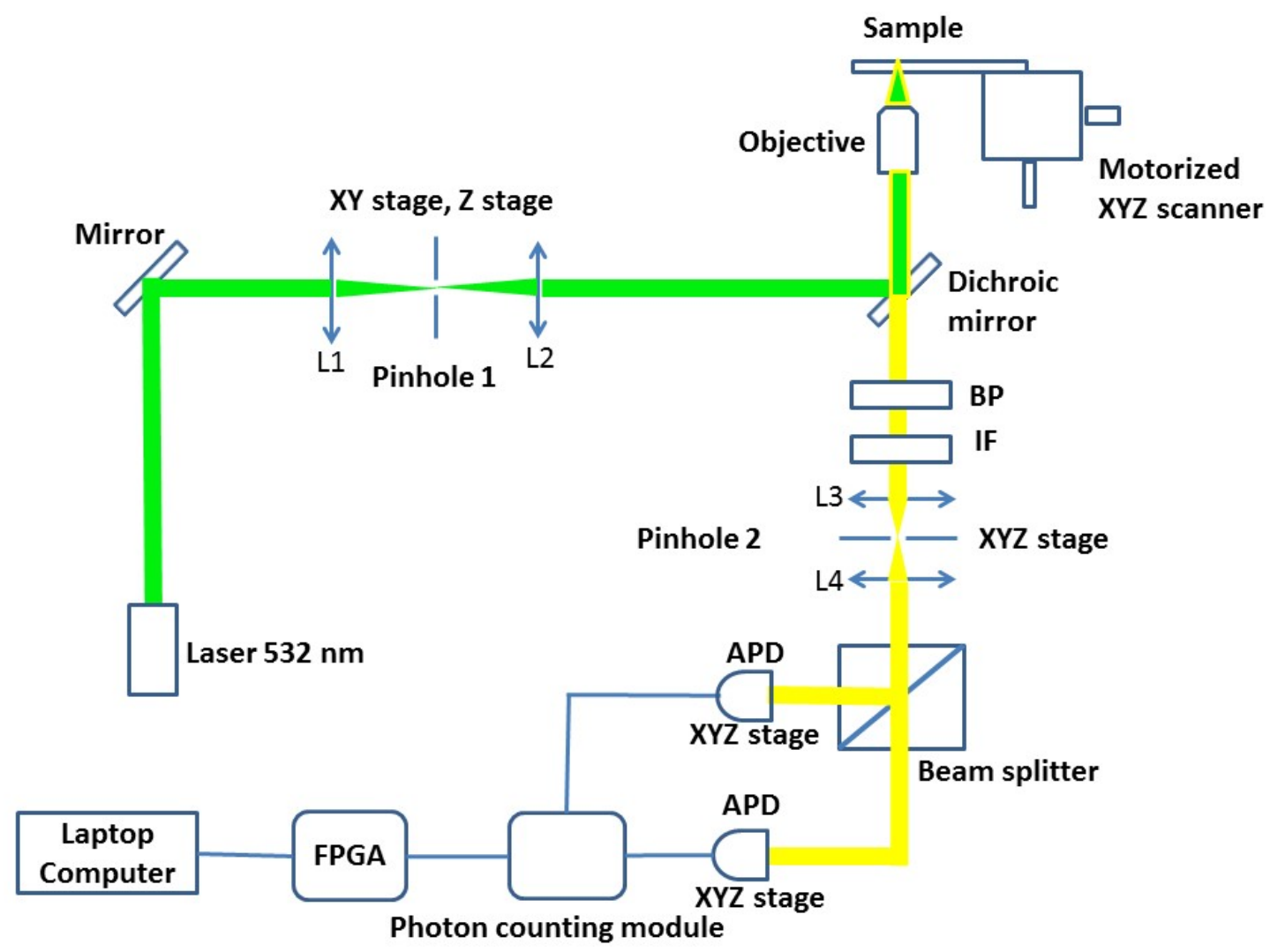

Fig. 2. Schematic sketch of the experiment setup to measure anti-bunching effect.

\section{EXPERIMENT PREPARATION}

In our measurements, the samples of dye molecules or quantum dots are prepared in distilled water with predetermined very dilute concentration and transferred to the thin cover slip of $0.13-0.17 \mathrm{~mm}$ in thickness using micropipettes. Coverslips are thoroughly cleaned and dried before the experiments. We use different neutral density filters ranging from $O D=0.5$ to 2 before the spatial filter to adjust the excitation laser power to suitable levels. The laser power after the spatial filter is measured using a Coherent power meter and shown to be in the range from 3 to $400 \mu \mathrm{W}$. Laser grade dye Rhodamine $\mathrm{B}$ is used for calibration purpose of the FCS instrument before taking the measurements. The same Rhodamine B and water soluble CdTe quantum dots made by PlasmaChem GmbH (Germany) which emit a wide spectrum peaking at wavelength of $600 \mathrm{~nm}$ are used to measure the antibunching effect. 


\section{MEASUREMENT RESULTS AND DISCUSSION}

The measurements of anti-bunching effect from dye molecules and quantum dot nanoparticles require several steps. We first carried out the calibration of instrument as outlined in Ref. [14]. Due to the use of much more sensitive APD detectors, the FCS correlation curves can be now measured with much higher signal to noise and in much less time. The sample solution is gradually diluted until the FCS data indicate that on average there is less than one or two molecules or quantum dots in the measurement volume as shown in Fig. 3. We then proceed to measure the $g^{(2)}(\tau)$ correlation function using the HBT configuration. The results for Rhodamine B and CdTe quantum dots are shown in Figs. 4 and 5. The measured $g^{(2)}(\tau)$ correlation functions with a bintime of $0.512 \mathrm{~ns}$ clearly indicate the presence of a large dip at a delay time $\tau=22 \mathrm{~ns}$. That delay time is due to the experimental setup with longer cable inserted between the APD and the photon counting module. The detection of a dip in the second order correlation function proves that dye molecule and CdTe quantum dot behave as an isolated two-level quantum system, which is entirely consistent with our expectation.

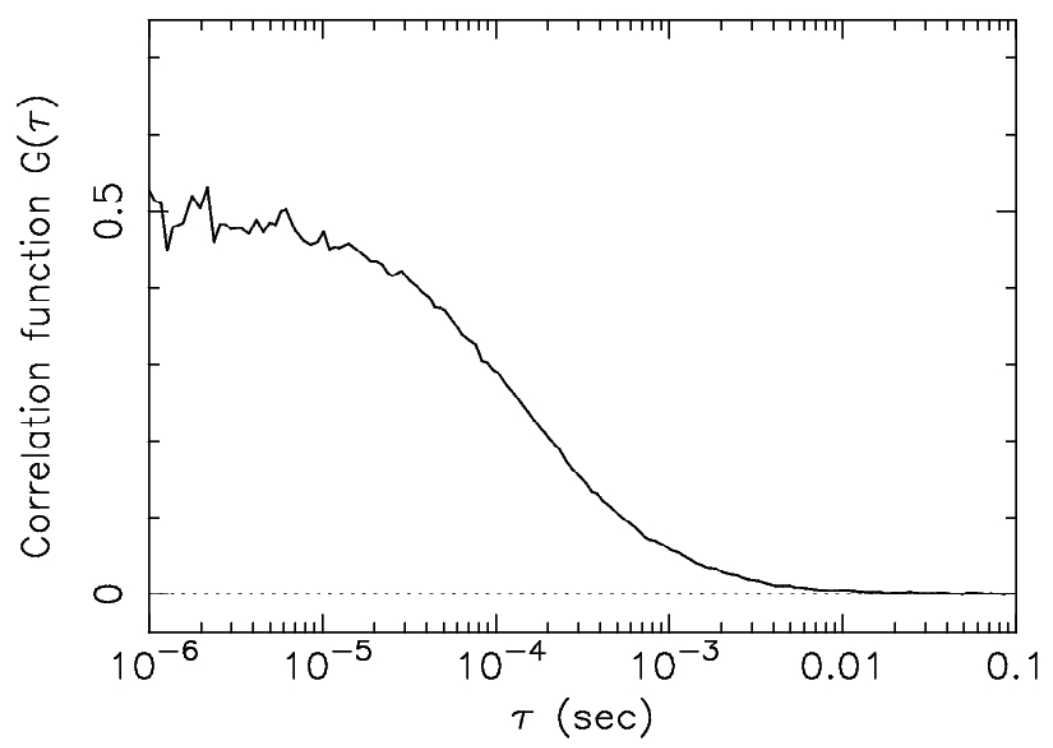

Fig. 3. Fluorescence correlation function of dilute solution of CdTe quantum dots measured with our experiment setup.

By matching the second order correlation function $g^{(2)}(\tau)$ with the form shown in Eq. (1), we derive the fluorescence lifetime of approximately $2 \mathrm{~ns}$ for Rhodamine B and about $45 \mathrm{~ns}$ for CdTe quantum dots as shown in Figs. 4 and 5. Our estimated fluorescence lifetime for Rhodamine $\mathrm{B}$ is consistent with recent measurement of fluorescence life time between $1.5-1.7 \mathrm{~ns}$ using time correlated single photon counting technique [15]. For CdTe quantum dots, the fluorescence lifetime is known to be in the range from a few nanoseconds to $\sim 40 \mathrm{~ns}$, depending on properties of quantum dots $[7,16]$. Therefore, our fitting result for the lifetime of CdTe quantum dots from PlasmaChem is consistent with previous measurements. 


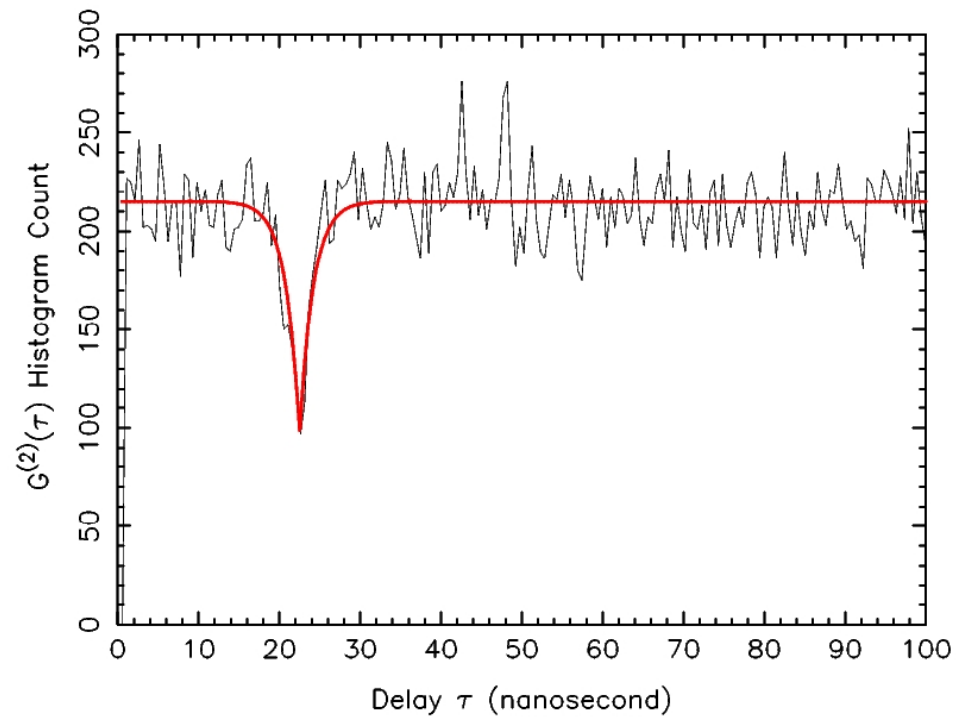

Fig. 4. The $g^{(2)}(\tau)$ correlation function from Rhodamine B molecules. Red thick solid line represents the best match between theoretical $g^{(2)}(\tau)$ function and the measurement data, corresponding to a lifetime of $2 \mathrm{~ns}$.

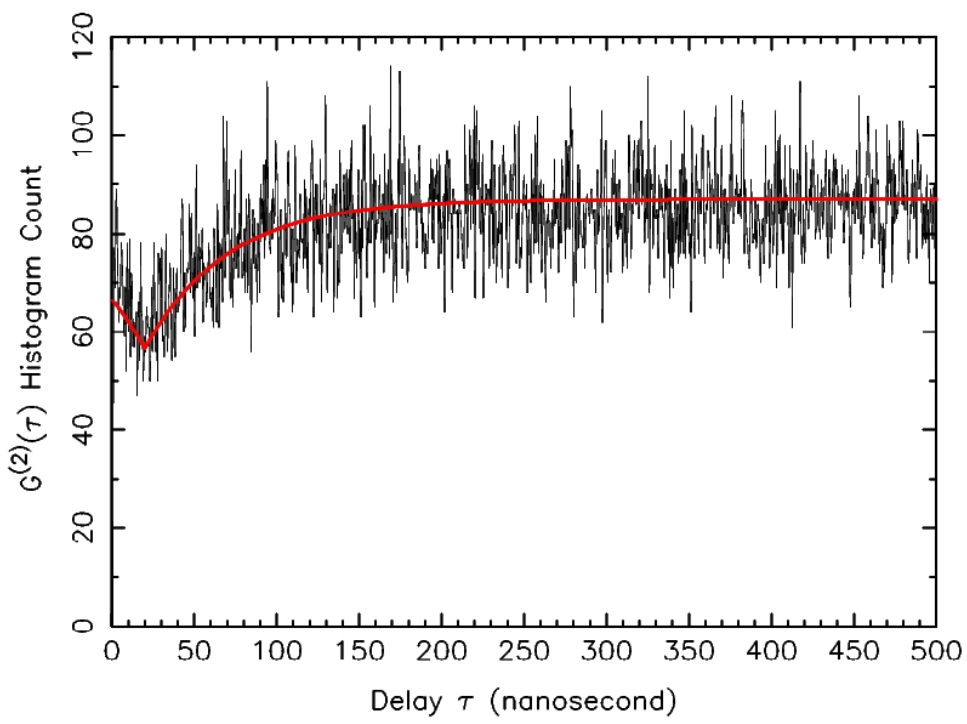

Fig. 5. The $\mathrm{g}^{(2)}(\tau)$ correlation function from CdTe quantum dot nanoparticles. Red thick solid line represents the best match between theoretical $\mathrm{g}^{(2)}(\tau)$ function and the measurement data, corresponding to a lifetime of $45 \mathrm{~ns}$.

The measurements of second order correlation function open a new and independent way to directly assess the fluorescence lifetime of single nanoparticle or single molecules without the 
use of the usual lifetime measurement methods such as time correlated single photon counting, which would require the use of ultrafast (picosecond or femtosecond) laser systems. These experiments mark the first time that we could measure directly quantum effect using locally developed instrumentation and, as a result, derive directly the fluorescence lifetime in nanosecond range.

\section{CONCLUSION}

In this paper we have detailed our experiment to measure the anti-bunching effect in the emission from single dye molecule Rhodamine B and CdTe quantum dot nanoparticles. We show that the antibunching curves can be fitted with the theoretical prediction and estimate the fluorescence lifetime of these single molecules or single nanoparticles as isolated quantum systems. The measurements open the way for deeper research into quantum optics and nanotechnology applications.

\section{ACKNOWLEDGEMENT}

We acknowledge the financial support of the Vietnam Academy of Science and Technology through project number VAST01.01/12-13.

\section{REFERENCES}

[1] S. Fore, T. A. Laurence, C. W. Hollars, and T. Huser, IEEE Journal of Selected Topics in Quantum Electronics 13 (4) (2007) 996-1005.

[2] D. Stoler, Physical Review Letters 33 (23) (1974) 1397.

[3] H. J. Kimble, M. Dagenais, and L. Mandel, Physical Review Letters 39 (11) (1977) 691.

[4] T. Basché, W. Moerner, M. Orrit, and H. Talon, Physical Review Letters 69 (10) (1992) 1516.

[5] B. Lounis and W. E. Moerner, Nature 407 (6803) (2000) 491-493.

[6] P. Michler, A. Imamoğlu, M. Mason, P. Carson, G. Strouse, and S. Buratto, Nature 406 (6799) (2000) 968-970.

[7] B. Lounis, H. Bechtel, D. Gerion, P. Alivisatos, and W. Moerner, Chemical Physics Letters 329 (5) (2000) 399404.

[8] I. König, A. Zarrine-Afsar, M. Aznauryan, A. Soranno, B. Wunderlich, F. Dingfelder, J. C. Stüber, A. Plückthun, D. Nettels, and B. Schuler, Nature Methods 12 (8) (2015) 773-779.

[9] K. D. Weston, M. Dyck, P. Tinnefeld, C. Müller, D. P. Herten, and M. Sauer, Analytical Chemistry 74 (20) (2002) 5342-5349.

[10] X.-W. Wu, M. Gong, C.-H. Dong, J.-M. Cui, Y. Yang, F.-W. Sun, G.-C. Guo, and Z.-F. Han, Optics Express 18 (6) (2010) 6340-6346.

[11] J. Sỳkora, K. Kaiser, I. Gregor, W. Bönigk, G. Schmalzing, and J. Enderlein, Analytical Chemistry 79 (11) (2007) 4040-4049.

[12] F. Rabouw, N. Cogan, A. Berends, S. Wv, D. Vanmaekelbergh, A. Koenderink, T. Krauss, et al., Scientific Reports 6 (2015) 21187-21187.

[13] S. Kitson, P. Jonsson, J. Rarity, and P. Tapster, Physical Review A 58 (1) (1998) 620.

[14] N. T. T. Bao and D. V. Trung, Communications in Physics 25 (1) (2015) 59.

[15] A. S. Kristoffersen, S. R. Erga, B. Hamre, and Ø. Frette, Journal of Fluorescence 24 (4) (2014) 1015-1024.

[16] M. Dahan, T. Laurence, F. Pinaud, D. Chemla, A. Alivisatos, M. Sauer, and S. Weiss, Optics Letters 26 (11) (2001) 825-827. 
